\title{
Comparison of structures among Saccharomyces cerevisiae Grxs proteins
}

Mohnad Abdalla $a^{1,2^{*+}}$ D, Wafa Ali Eltay $b^{2,3^{*}+}$ and Aadil Yousif ${ }^{4}$

\begin{abstract}
Glutaredoxins (Grxs) comprise a group of glutathione (GSH)-dependent oxidoreductase enzymes that respond to oxidative stress and sustain redox homeostasis. Saccharomyces cerevisiae Grx has a similar interaction patterns through its residues between the residues and the environment. The glutaredoxin domain covers $100 \%$ of the entire mature Grx1 and Grx8, while the glutaredoxin domain covers 52\% of the entire mature Grx6 and Grx7, which have approximately 74 additional amino acids in their N-terminal regions, whereas Grx3 and Grx4 have two functional domains: glutaredoxin and thioredoxin. We have presented the prediction of disordered regions within these protein sequences. Multiple sequence alignment combined with a phylogenetic tree enabled us to specify the key residues contributing to the differences between Saccharomyces cerevisiae Grxs and the proportion symmetry.
\end{abstract}

Keywords: Grx, Saccharomyces cerevisiae, Protein-protein interactions, Domain, Disorder, Transmembrane

\section{Background}

The Glutaredoxin family (Grxs) has been portrayed as a part of the thioredoxin-fold oxidoreductase superfamily, which have a catalytic centre with a highly conserved CXXC/S motif. Grxs contribute to various biological functions, including protection against the oxidative damage induced by several reactive oxygen species (ROS) [1], as well as iron-sulfur cluster assembly in reaction to cellular iron availability [2]. Grxs stimulate the reduction of mixed disulfides through low-molecular-weight thiols together with reduced glutathione (GSH) or other proteins in the presence of GSH, NADPH, and glutathione reductase [3]. It has been shown that to lower the $\mathrm{pK}_{\mathrm{a}}$ value, the $\mathrm{N}$-terminus cysteine of Grxs should be leaving group in the catalytic reaction with a good nucleophile $[4,5]$. Grxs can be classified into dithiol and monothiol. Grxs depend on the number of cysteine residues in the active-site of the CXXC/S motif [6-8]. The conserved active-site motifs are usually CPYC in the classical dithiol Grxs and CGFS in the monothiol Grxs. Generally, members of the Grx family are able to bind to GSH through hydrogen bonds in addition

\footnotetext{
*Correspondence: mohnadabdalla200@gmail.com; wafa.ali.11338@gmail.com ${ }^{+}$Mohnad Abdalla and Wafa Ali Eltayb contributed equally to this work. ${ }^{1}$ Faculty of Science and Technology, Omdurman Islamic University, Omdurman, Sudan

${ }^{2}$ School of Life Sciences, University of Science and Technology of China, Hefei, Anhui 230027, People's Republic of China

Full list of author information is available at the end of the article
}

to mixed disulfide bonds, as well as electrostatic and highly hydrophobic interactions [9-11].

The kinetic test proposed that Grxs may have two catalytic mechanisms: sequential and ping-pong [12]. The crystal structures of yeast Grx1, Grx2, Grx5, Grx6, and Grx8 have been solved and their enzymatic activities compared. These analyses showed the diversity of Grxs which are encoded by the same organism from both catalytic and structural points of view [13-16].

\section{Protein-protein interactions network}

In a living cell, proteins do not work as single entities, but they form an assortment of functional connections with each other, which are essential in cellular processes. To define how Grxs interact with other host protein families and affect cell functions, the proteins were identified and analysed by searching the STRING database. The STRING database is a tool for the retrieval of functional protein/protein and gene/protein interactions, as well as to determine the probable biological processes affected. The protein interaction network of the Grxs protein was specified using the STRING database [17]. Analysis of protein-protein interactions was performed to identify functionally linked proteins and determine the biological processes that were potentially affected by the known and predicted Grxs interaction partner network. The confidence view screenshot from the STRING 
database (Search Tool for the Retrieval of Interacting Genes/Proteins) shows the known and predicted protein-protein interaction network of Saccharomyces cerevisiae Grx6, which was used as the input for the query. Stronger associations are represented by thicker lines. The required high confidence score was limited to over $75 \%$, and the protein-protein interaction network was limited to the 10 best-scoring hits. The interaction network showed the association between differentially expressed proteins. The interaction map was generated using default settings (high confidence of 0.7 and 7 criteria for linkage: neighbourhood, gene fusion, co-occurrence, co-expression, experimental evidence, existing databases and text mining). Based on its literature value and database, it created the interactions with the protein of interest. Using the STRING database to create protein interactions is very helpful to understand the protein because it shows which proteins interact with Grx6.

The Saccharomyces cerevisiae Grxs interaction network shows that Grxs proteins form a highly complex, exceedingly organized network of interacting proteins as expected, as shown by the search tool for the Grxs of interacting proteins and/or genes (STRING) network analysis. Most identified proteins show main functional involvement. Clusters which contain thioredoxin isoenzyme (TRX1 and TRX2 and TRX3) [18], thioredoxin reductase (TRR1), methionine-R-sulfoxide reductase (MXR2), mitochondrial peroxiredoxin (PRX1), thiol peroxidase (HYR1), $\mathrm{NAD}(+)$-dependent glutamate synthase (GLT1) [19, 20], and protein kinase (BUD32) are involved in the negative regulation of the transcription of iron regulon (FRA2) and proteins of unknown function (YAL044W-A, YDL009C, YDL057W). Activator of ferrous transport (AFT1) is a transcription factor involved in iron utilization and homeostasis [21]. FRA2 is a protein involved in the negative regulation of transcription of iron regulon $[22,23]$. BolA-like protein (AIM1) is a protein involved in mitochondrial function and organization. Until now literature review showed that both Grx3 and Grx4 interacted with AFT1 to form hetero-oligomers [24]. However, there has been no information made available about an additional interaction. Analysis of the activity and expression of these

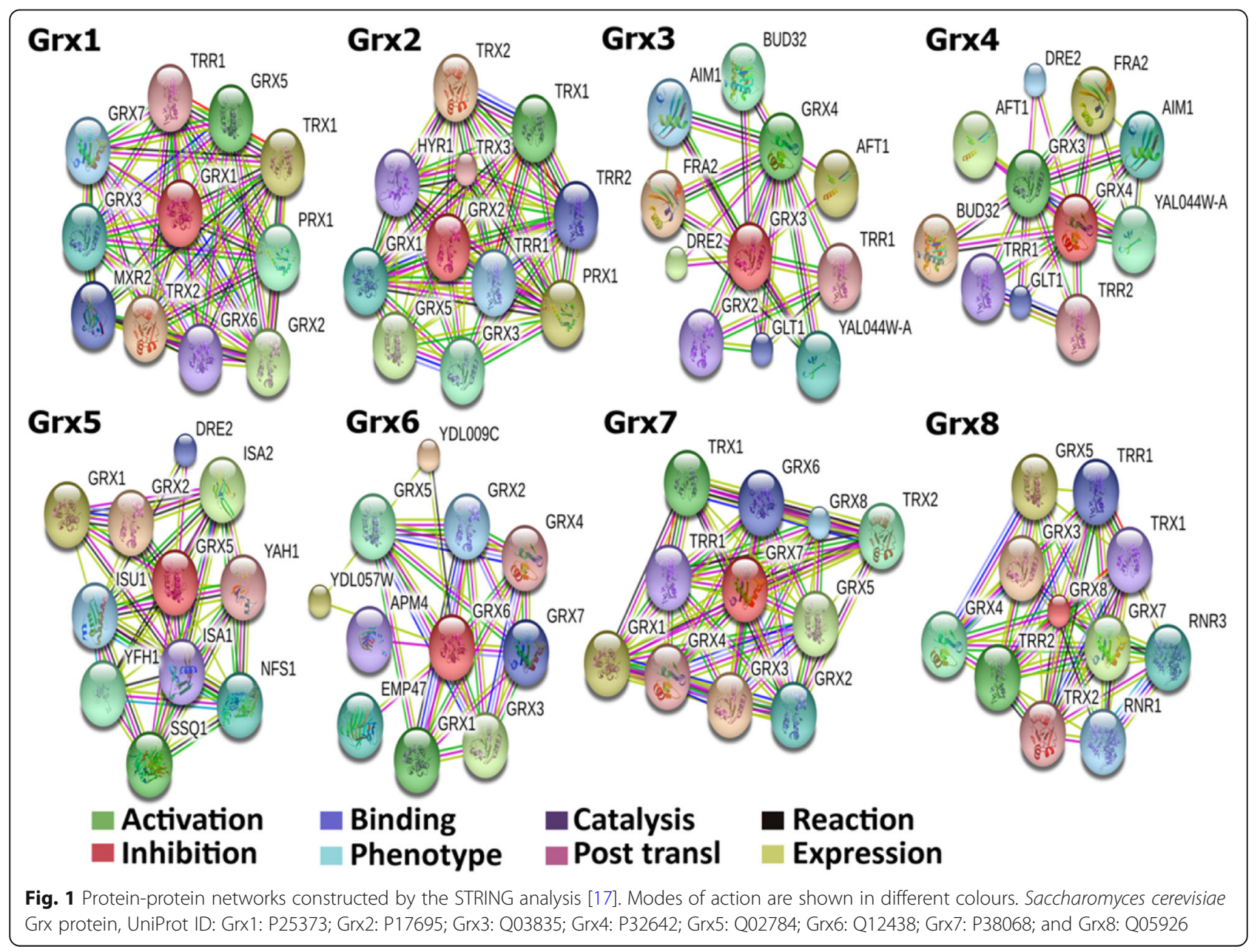


directly associating proteins in Saccharomyces cerevisiae may provide insights and thus is a good avenue for future study (Fig. 1).

\section{Grx domain}

Grx is a small, single or multi-domain (Fig. 2) protein. Analysis of the protein domain organization showed that the glutaredoxin domain covers $100 \%$ of the entire mature Grx1 [14] and Grx8 [15], while the glutaredoxin domain covers $\sim 52 \%$ of the entire mature Grx6 [6] and Grx7, which have an additional approximately 74 amino acids in their N-terminal regions. In contrast, Grx3 and Grx4 have two functional domains, glutaredoxin and thioredoxin. Glutaredoxin represents a proportion of $\sim 40 \%$, while thioredoxin represents a proportion of $\sim 46 \%$ of the entire mature protein, Grx2 glutaredoxin represents the proportions of 75\% [14] and Grx5 glutaredoxin represents the proportions of $\sim 68 \%$. Low-complexity regions (LCRs) are amino acid sequences that contain repeats of single amino acids or short amino acid motifs.

\section{Grx disorder}

Grxs also contain disordered regions, while they have structured regions. However, only Grx7 is almost fully disordered in its native state. From the disorder prediction we can see that the large disordered region in Grx3 is between residues 112 and 154, in Grx4 it is between residues 114 and 147, in Grx5 it is between 130 and 146, and there are two disordered regions in Grx6 between residues 42 and 85 and between residues 225 and 231 . Grx7 also has two disordered regions, between 35 and 89 as well as between 129 and 172 . The Grx7 sequences consist of many random coil conformations with only a small ratio of helices and sheets. The large disordered region is located in the $\mathrm{C}$ domain in Grx3 and Grx4, while it is located in the $\mathrm{N}$ domain in Grx6, and in Grx7

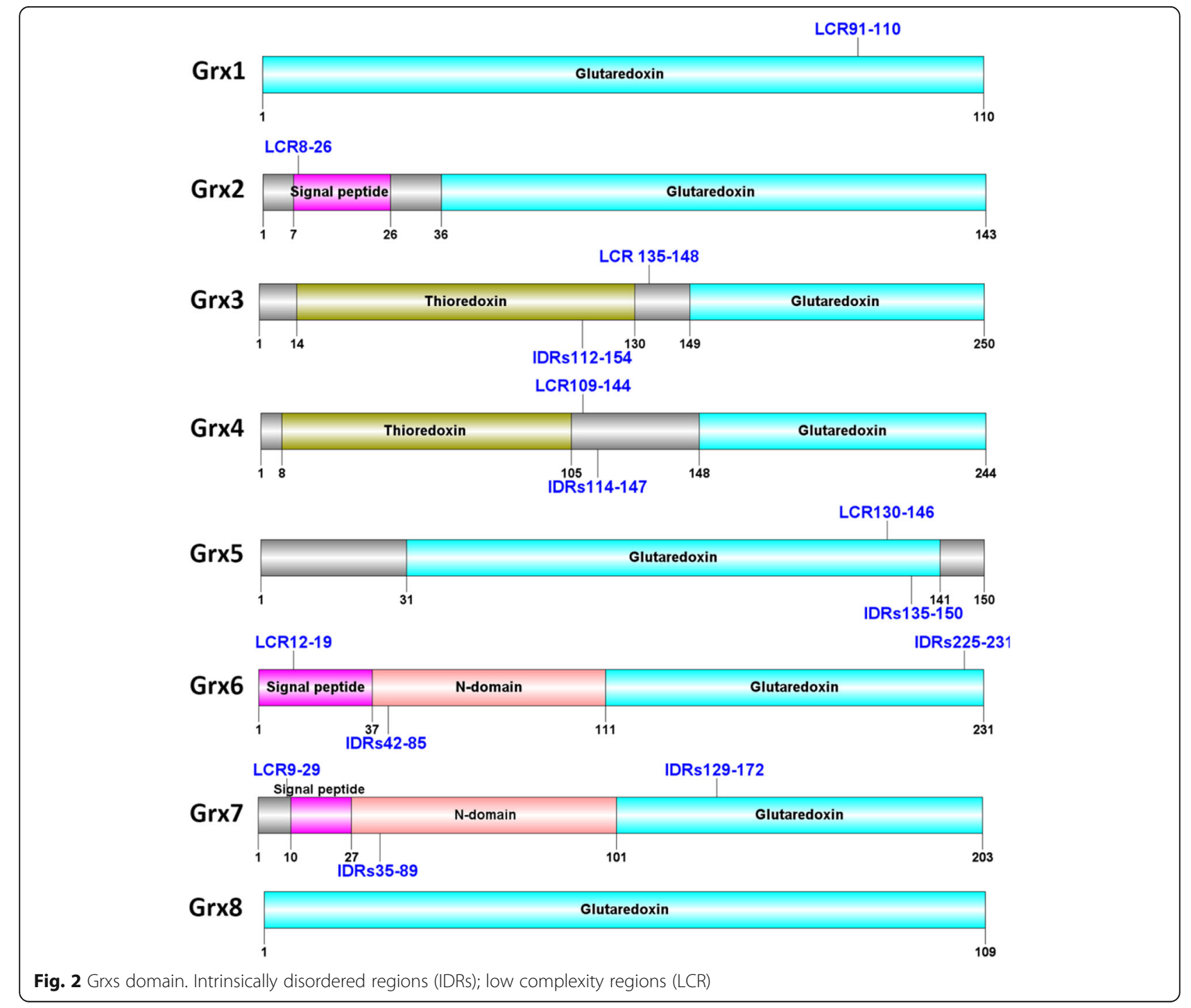




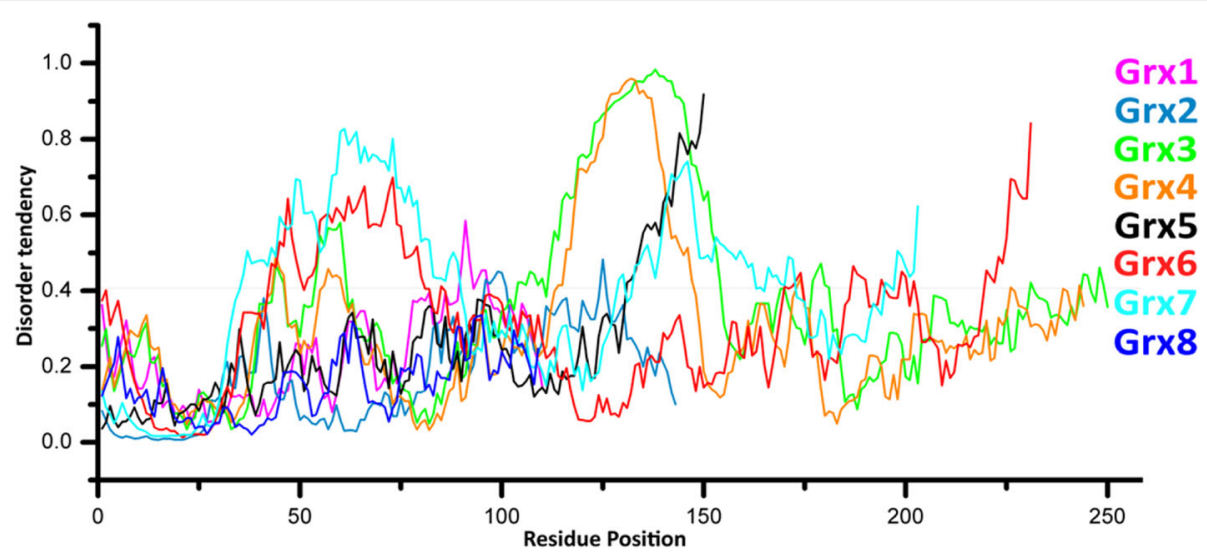

Fig. 3 Disorder profiles of Grx 1-8 sequences. Values above 0.4 are regarded as disordered. The data was obtained from the IUPred2A [25]

the disordered regions are located at both the $\mathrm{N}$ and $\mathrm{C}$ domains. In fact, the large disordered region loops with a high degree of mobility. Because of this, until now we were not able to determine the three-dimensional folded structures of Grx3, Grx4, Grx7, and the N domain of Grx6. Grx1, Grx2, Grx5, Grx6 C domain and Grx8 do not contain a large disordered region. The Saccharomyces cerevisiae Grxs are classified to three categories: structured, structured but containing a disordered region, and almost fully disordered. In addition, Grxs contain low complexity regions: Grx1 91-110, Grx2 8-26, Grx3 135-148, Grx4 109-144, Grx5 130-146, Grx6 1219 and Grx7 9-29. Numerous disordered regions imply low complexity sequences, and although low complexity sequences are a strong sign of disorder (Fig. 2), the inverse is not necessarily correct. Many LCRs are highly unstable due to replication slippage and recombination. However, not all disordered regions have low complexity sequences. Disordered regions have a low utility for the prediction of secondary structure. Sometimes, Grx sequences sustain a transformation from disorder to order upon interacting with ligands (GSH), such as the small disordered region in Grx6 225 to 231 (Fig. 3). The data was obtained from the IUPred2A [25].

\section{Grx superimposed}

Five structures are available among the eight solved to date. In general, the 3D structures of the Grxs superfamily are quite similar. Similarity between the structures is seen in Saccharomyces cerevisiae Grxs with 4 beta strands, except Grx6 with 6 beta strands. To assess how Grxs structural diversity, a 3D structure alignment was performed by the PyMOL 2.1 [26].

Grx6-314n consists of $6 \alpha$ helices and $6 \beta$ strands, while Grx6-5j3r consists of $5 \alpha$ helices and $6 \beta$ strands. That means the monomer has one more $\alpha$ helix, with the extra $\alpha$ helix situated between $\beta 2$ and $\alpha 4$. Leu125 and Ser126, which are part of $\alpha 1$ in $5 j 3$ r, are located in the loop between $\alpha 1$ and $\beta 1$ in $314 n$. The RMSD (root mean square deviation) between Grx6-5j3r and Grx6-314n is $0.37 \AA$.

There are slight conformational changes in Grx2-3ctg and Grx2-3ctf at the active site as well as conformational rearrangements between the residues Thr59 and Lys65. However, the major conformational change is $10.91 \AA$ at Tyr60 when it is bound to GSH [14]. The RMSD between Grx2-3ctg and Grx2-3ctf is $0.3 \AA$. Grx2-3ctg Leu138-Gln143 are located at the end of the C-terminus as a loop, but in 3ctf this amino acid it is part of $\alpha 6$. Gln50 and leu51 are located in the loop between $\alpha 1$ and

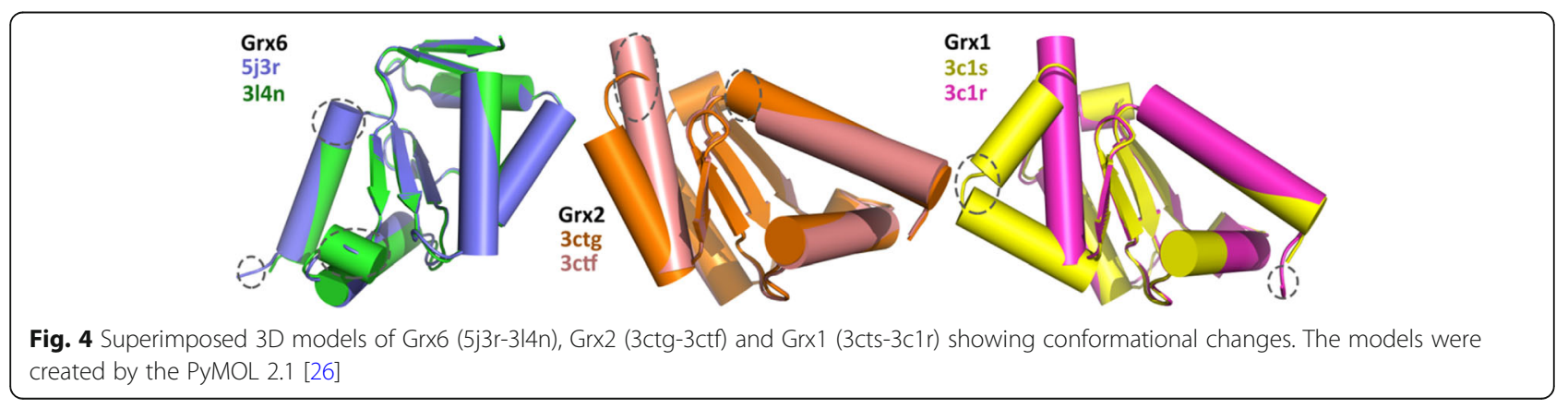




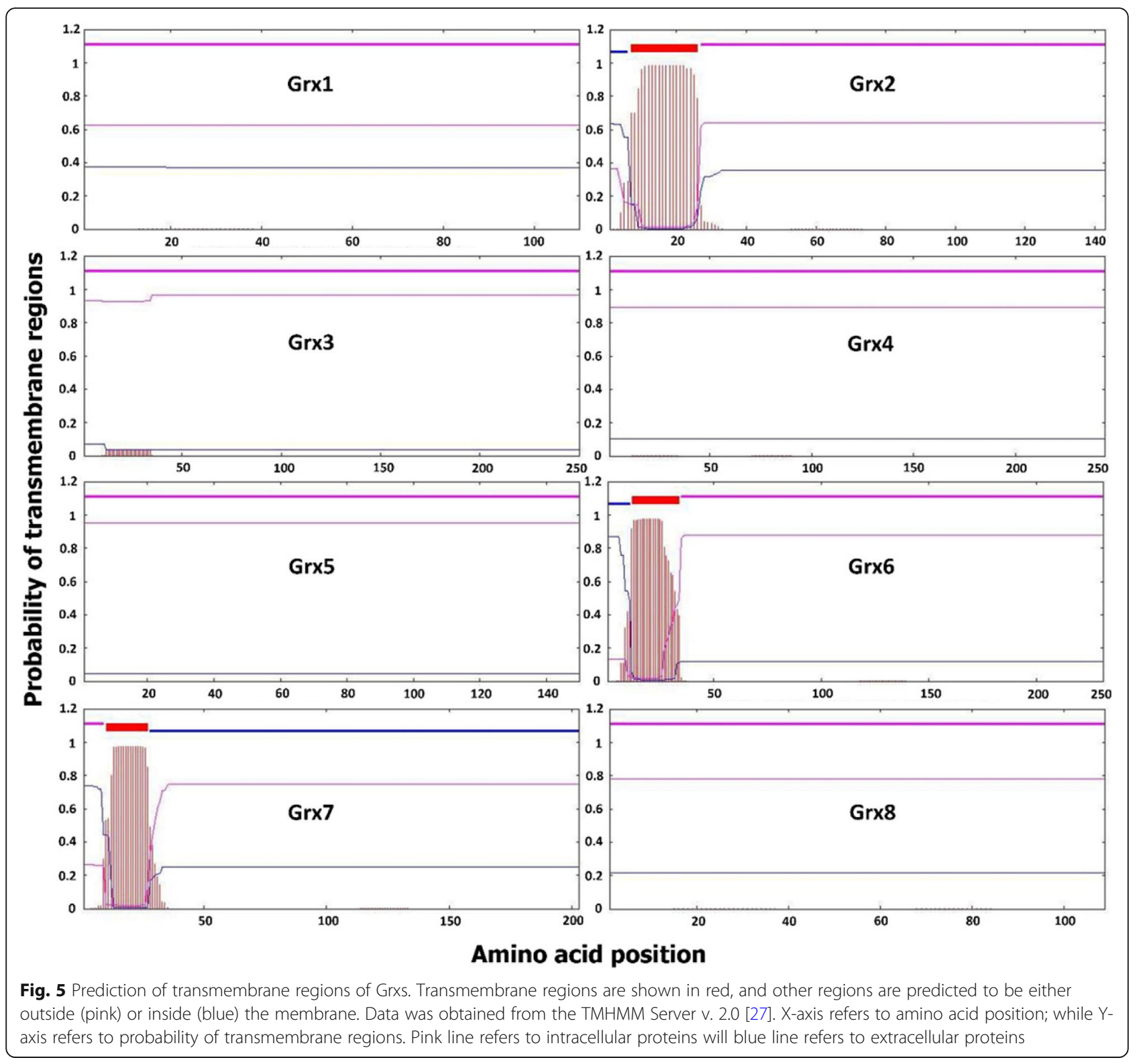

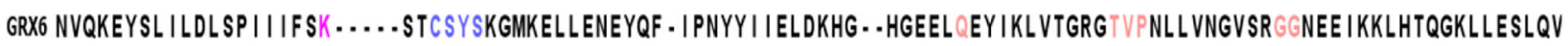
GRX1 - TIKHVKDLI AENE IFVASK …..TYCPYCHAALNTLFEKLKVPRSKVLVLQLNDMK . - EGAD I AAALYE INGQR TVPNIYINGKHI GGNDDLQELRETGELEELLEP GRX2 - TVAHVKDLI GQKEVFVAAK .....TYCPYCKATLSTLFQELNVPKSKALVLELDEMS - NGSE I DALEE I SGQKTVPNVYINGKHIGGNSDLETLKKNGKLAEILKP GRX3 QINARL TKLVNAAPVMLFMKGSPSEPKCGF SRQLVGILREHQV....RFGFFD...IL..RDESV QNLKKFSEWPTF PQLYINGEFQGGLDIIKESLEE..DPDFLQ.

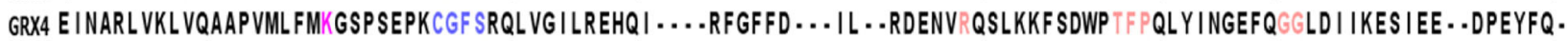

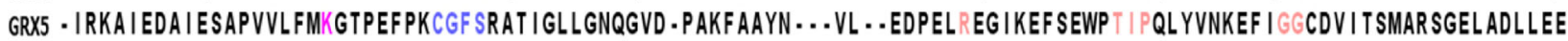
GRX7 DAAAEYNK IMEQSPMI IF SK ......TGCPYSKKLKALLTNSYTF -SPSYYVVELDRHE..HTKEL ODQIEKVTGRRTVPNVI IGGTSRGYYTEIAELHKNDELLDSFKK GRX8 ......AEEMIKSHPYFQLSA ......SWCPDCVYA-NS IWNKLNV-QDKVFVFDIGSLPRNEQEKWR I AFQKVVGSR NLPTIVVNGKFWGTESQLHRFEAKGTLEEELTK Fig. 6 Saccharomyces cerevisiae Grxs multiple sequence alignment. The alignment was created by the Clustal W [29]
} 


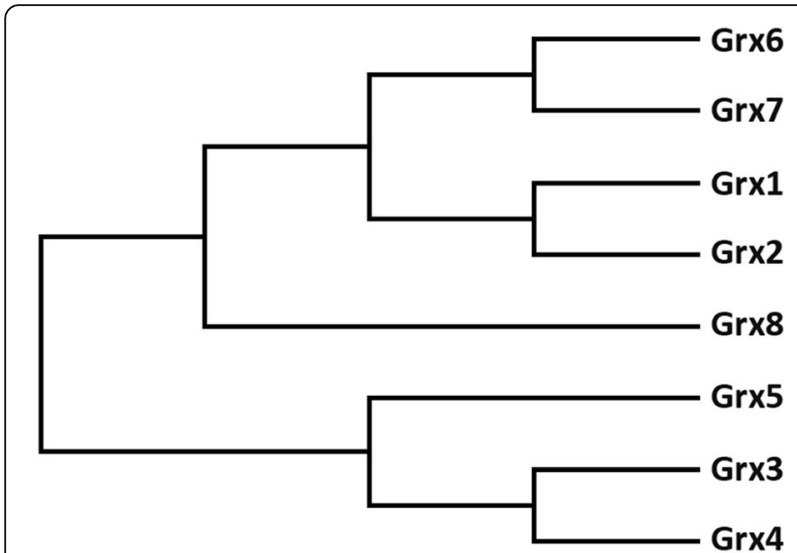

Fig. 7 Phylogenetic dendrogram of Saccharomyces cerevisiae Grxs. The phylogenetic tree derived from the Clustul W multiple sequence alignment was constructed by the maximum parsimony method of the MEGA7 software [30] $\beta 1$ in 3ctf, while there is a part of $\alpha 1$ in 3ctg. Grx2-3ctg and Grx2-3ctf consist of $6 \alpha$ and $4 \beta$.

There are dramatic conformational changes in Grx1-3c1s and Grx1-3c1r at the active site as well as conformational rearrangements in Thr25 and Tyr26. However, the major conformational change is $12.22 \AA$ at Tyr26 in the glutathionylated form. Gly98 in Grx1-3c1s is located in the loop between $\alpha 6$ and $\alpha 7$, while it is located in $\alpha 5$ at 3c1r. In addition, $\alpha 6$ in Grx1-3c1r is longer than $\alpha 7$ in Grx1-3c1s, $\alpha 6$ in Grx1-3c1r between Gly98 and Ala109, and $\alpha 7$ in Grx1-3c1s between Glu99 and Glu105. This means that conformational changes of Grx depend on the type of ligand. Grx1-3c1s is an amino acid involved in GSH-forming hydrogen bonds with (Asp89-Asn88-Cys27-Val75-Lys24-Gln63). These interactions are also determined in other Grx complexes with GSH, while two water molecule-mediated hydrogen bonds exist between (Asp89-Asn88) and MES in Grx1-3c1r. The RMSD between Grx1-3c1s and Grx1-3c1r RMSD is $0.51 \AA$. Grx1-3c1s consists of $7 \alpha$ helices and $4 \beta$ strands, while Grx1-3c1r consists of $6 \alpha$ helices and $4 \beta$ strands (Fig. 4).

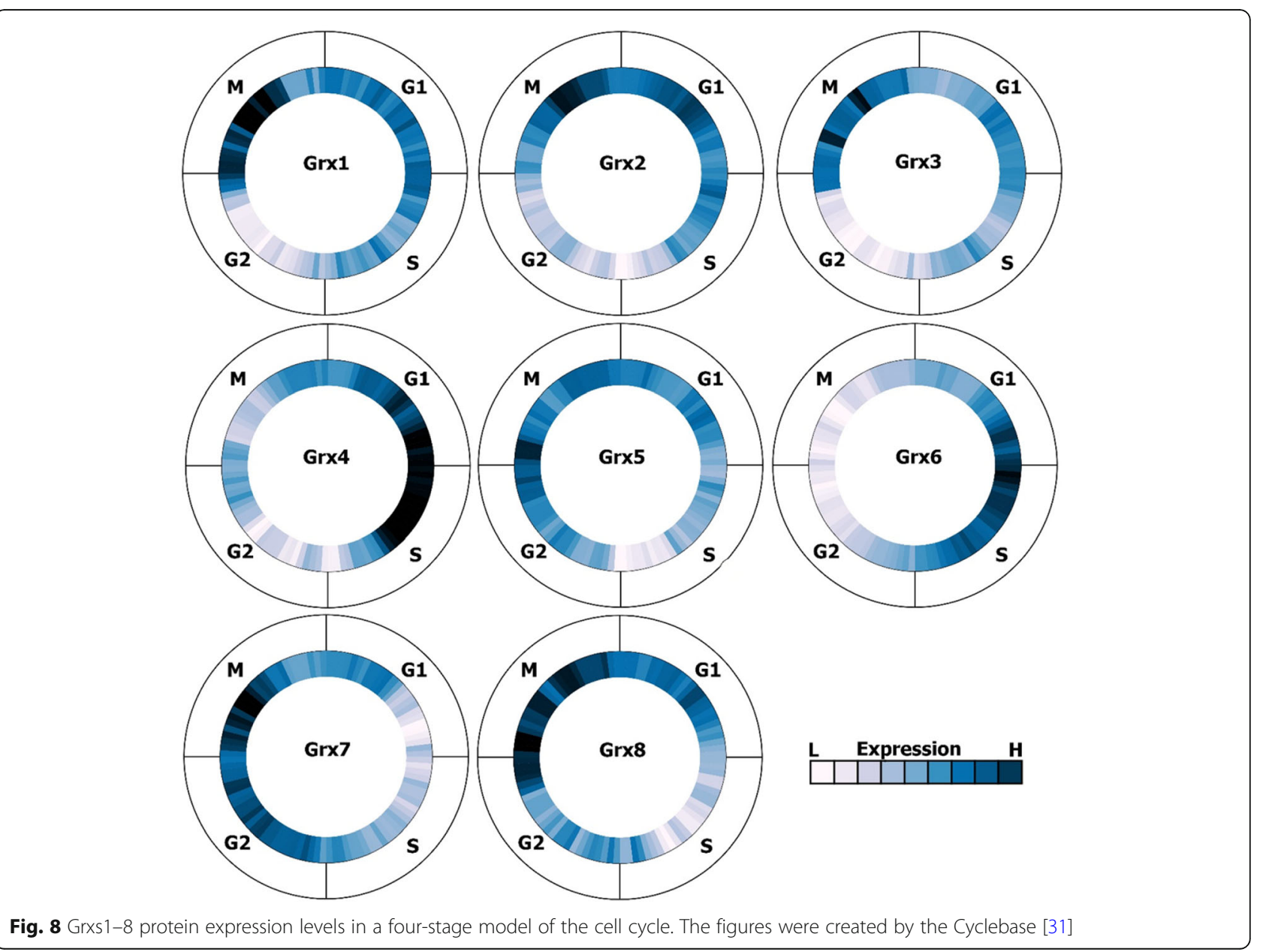




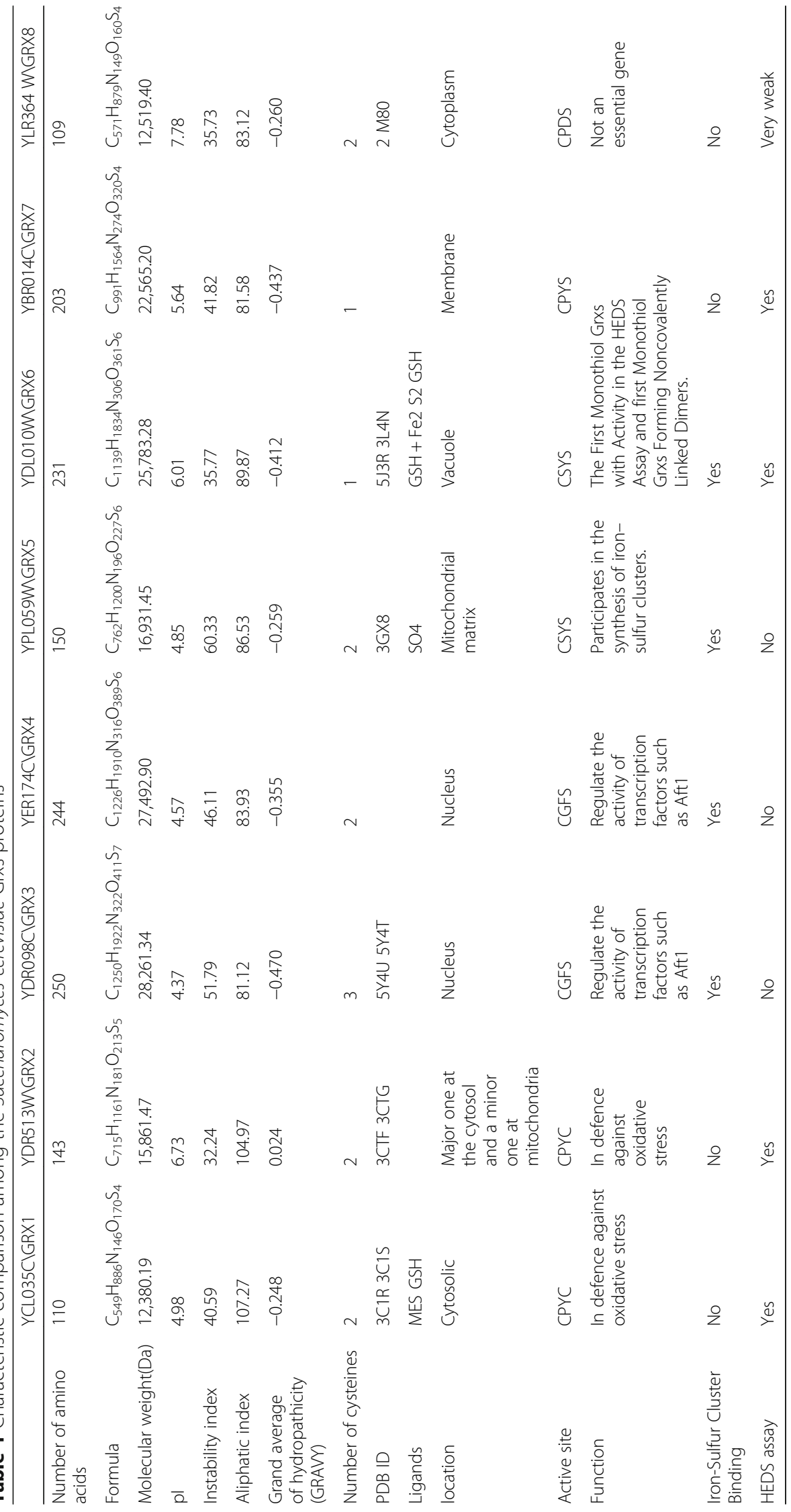


Grx proteins are constantly changing their conformation in both their oxidized and reduced forms. It is not clear whether these slight differences have an important impact on substrate selectivity or protein function.

\section{Grx transmembrane}

Single protein regions are known to have various types of functions. One of these functions leads to the translocation of the polypeptide into the endoplasmic reticulum, which is very important to identify. We therefore analysed the transmembrane regions [27]. Grxs transmembrane regions were found only in Grx2, Grx6 [28], and Grx7. Signal peptide prediction showed only one signal peptide in each of them, and all of them localized at the N-terminus (Fig. 5). All Saccharomyces cerevisiae Grxs are predicted as intracellular proteins except that Grx7 was predicted as extracellular protein (Fig. 5). The existence of a signal sequence and a disorder sequence also backs up this localization. Additionally, this localization has not been demonstrated before and gives us extra details about the of Saccharomyces cerevisiae cell wall architecture.

\section{Grxs multiple sequence alignment}

Highlighted are a number of structural similarities and conserved motifs that are common to plants, animals, humans, and bacteria. However, one or two differences may affect protein function. The deduced amino acid sequences of Grx comprised of a conserved TVP and GG at the $\mathrm{C}$ domain involves GSH binding. TVP and GG are the second most commonly matched sequence in the Grxs superfamily, after the active site (Fig. 6). However, despite the sequence similarity, there are some crucial differences in the active site architectures. The one cysteine residue is extremely well conserved, and the Grxs active sites have similar biochemical properties. Information about the biochemical and/or biological properties is shown in Table 1.

The key modifications in Grxs are at the active-site CXXC motif and at the GSH-recognition site. Grx8 utilizes a CPDC motif instead of the $\mathrm{CPY}(\mathrm{F}) \mathrm{C}$ motif found in other Grxs. Moreover, the TVP motif in almost all Grxs is involved in the bond with the cysteine moiety of GSH. The Gln residue is implicated in stabilizing the glycine moiety of GSH.

\section{The phylogeny of Grx}

The yeast Saccharomyces cerevisiae encodes ten Grxs, eight of which have been characterized, including five monothiol Grxs and three dithiol Grxs. There is a high degree of similarity shared between the Grx1 and Grx2 sequences (64\%), the Grx3 and Grx4 sequences (66.8\%), and the Grx6 and Grx7 sequences (37.5\%). A phylogenetic tree
(Fig. 7) which was constructed for the validated Saccharomyces cerevisiae Grxs reveals that Grx1 and Grx2, Grx3 and Grx4, and Grx6 and Grx7 well-defined to be more closely related to each other than to Grx5 and Grx8. However, there are some differences among Grx1, Grx2, Grx6 and Grx7 in GSH-dependent oxidoreductase activity because of their slightly different in the active-site structures. Closely related pairs include Grx1 and Grx2, Grx3 and Grx4, and Grx6 and Grx7.

\section{Grx protein expression levels}

The prediction shows that Grx4 possesses the highest level of the expression and longest period of expression compared to other Grxs. Grx6 expression stage is almost opposite to Grx7. Grx6 increases its level of expression between the end of stage G1 and the middle stage of S, while Grx7 increases its level of expression between the middle stage of stage $\mathrm{G} 2$ and the middle stage of $\mathrm{M}$. However, Grx1 and Grx2 have very low expression in the G2 stage, while the expression increases for both of them in stage M. Furthermore, Grx3 increases its level of expression in stage $M$, while Grx4 possesses a low level of expression in the G1 stage (Fig. 8).

\section{Conclusion}

Due to the sequence conservation, overlapping protein interactions, and matching of disordered profile, glutaredoxin domain of a similar length in eight proteins leads to similar functions. It is clear from this analysis that a considerable number of amino acid sequences represent a random coil configuration in Grx7, leading to an inability to solve the structure. Eight proteins have almost same interaction partner as the Grx protein with high similarly and conservation.

\section{Abbreviations \\ Cys: Cysteine; ER: Endoplasmic reticulum; Fe/S: Iron-Sulfur; Grx: Glutaredoxin; GSH: Glutathione; PDB: Protein data bank}

Acknowledgements

We thank Chinese Academy of Sciences for their support.

Funding

Not applicable.

Availability of data and materials

Not applicable.

Authors' contributions

MA and WAE analyzed the data and wrote the manuscript, MA WAE, AY read and approved the final manuscript.

\section{Authors' information}

Mohnad Abdalla: mohnadabdalla200@gmail.com

Hefei National Laboratory for Physical Sciences at the Microscale. Wafa Ali Eltayb: wafa.ali.113388@gmail.com

Hefei National Laboratory for Physical Sciences at the Microscale. Aadil Yousif: aadilyousif@yahoo.com

Faculty of Applied Medical Sciences, Tabuk University, Saudi Arabia. 


\section{Ethics approval and consent to participate}

Not applicable.

\section{Consent for publication}

Not applicable.

\section{Competing interests}

The authors declare that they have no competing interests.

\section{Publisher's Note}

Springer Nature remains neutral with regard to jurisdictional claims in published maps and institutional affiliations.

\section{Author details}

'Faculty of Science and Technology, Omdurman Islamic University, Omdurman, Sudan. ${ }^{2}$ School of Life Sciences, University of Science and Technology of China, Hefei, Anhui 230027, People's Republic of China. ${ }^{3}$ Faculty of Science and Technology, Shendi University, Shendi, Nher Anile, Sudan. ${ }^{4}$ Faculty of Applied Medical Sciences, University of Tabuk, Tabuk, Saudi Arabia.

\section{Received: 9 April 2018 Accepted: 12 July 2018}

Published online: 03 September 2018

\section{References}

1. Luikenhuis S, Perrone G, Dawes IW, Grant CM. The yeast Saccharomyces cerevisiae contains two glutaredoxin genes that are required for protection against reactive oxygen species. Mol Biol Cell. 1998;9(5):1081-91. Available from: https://www.ncbi.nlm.nih.gov/pmc/articles/PMC25331/

2. Li H, Mapolelo DT, Dingra NN, Naik SG, Lees NS, Hoffman BM, Riggs-Gelasco PJ, Huynh BH, Johnson MK, Outten CE. The yeast iron regulatory proteins Grx3/4 and Fra2 form heterodimeric complexes containing a [2Fe-2S] cluster with cysteinyl and histidyl ligation. Biochemistry. 2009;48(40):956981. Available from: https://www.ncbi.nlm.nih.gov/pubmed/19715344

3. Holmgren A, Aslund F. Glutaredoxin. Methods Enzymol. 1995;252:283-92. Available from: https://www.ncbi.n/m.nih.gov/pubmed/7476363

4. Gallogly MM, Starke DW, Leonberg AK, Ospina SM, Mieyal JJ. Kinetic and mechanistic characterization and versatile catalytic properties of mammalian glutaredoxin 2: implications for intracellular roles. Biochemistry. 2008;47(42): 11144-57. Available from: https://www.ncbi.nlm.nih.gov/pubmed/18816065

5. Gallogly MM, Starke DW, Mieyal JJ. Mechanistic and kinetic details of catalysis of thiol-disulfide exchange by glutaredoxins and potential mechanisms of regulation. Antioxid Redox Signal. 2009;11(5):1059-81. Available from: https://www.ncbi.nlm.nih.gov/pmc/articles/PMC2842129

6. Abdalla M, Dai YN, Chi CB, Cheng W, Cao DD, Zhou K, Ali W, Chen Y, Zhou CZ. Crystal structure of yeast monothiol glutaredoxin Grx6 in complex with a glutathione-coordinated [2Fe-2S] cluster. Acta Crystallogr F Structural Biol Commun. 2016;72(Pt 10):732-7. Available from: https://www.ncbi.nlm.nih. gov/pubmed/27710937

7. Eltayb WA, Abdalla M, Samad A, EL-Arabey AA, Ghanam AR, Almahi WA. Number of cysteine interactions with the activity in GRX family. J Proteomics Bioinform. 2017;10(4):114-8. Available from: https://www. omicsonline.org/peer-reviewed/number-of-cysteine-interactions-with-theactivity-in-grx-family-87704.html

8. Rouhier N, Couturier J, Johnson MK, Jacquot JP. Glutaredoxins: roles in iron homeostasis. Trends Biochem Sci. 2010;35(1):43-52. Available from: https:// europepmc.org/abstract/med/19811920

9. Nordstrand K, Slund F, Holmgren A, Otting G, Berndt KD. NMR structure of Escherichia coli glutaredoxin 3-glutathione mixed disulfide complex: implications for the enzymatic mechanism. J Mol Biol. 1999;286(2):541-52. Available from: http://europepmc.org/abstract/MED/9973569

10. Yu J, Zhang NN, Yin PD, Cui PX, Zhou CZ. Glutathionylation-triggered conformational changes of glutaredoxin Grx 1 from the yeast Saccharomyces cerevisiae. Proteins. 2008;72(3):1077-83. Available from: https://onlinelibrary.wiley.com/doi/abs/10.1002/prot.22096

11. Yang Y, Jao S, Nanduri S, Starke DW, Mieyal JJ, Qin J. Reactivity of the human thioltransferase (glutaredoxin) C7S, C25S, C78S, C82S mutant and NMR solution structure of its glutathionyl mixed disulfide intermediate reflect catalytic specificity. Biochemistry. 1998;37(49):17145-56. Available from: https://www.ncbi.nlm.nih.gov/pubmed/9860827
12. Zaffagnini M, Bedhomme M, Marchand $\mathrm{CH}$, Couturier JR, Gao XH, Rouhier N, Trost P, Lemaire SP. Glutaredoxin s12: unique properties for redox signaling. Antioxid Redox Signal. 2012;16(1):17-32. Available from: https://www.ncbi. nlm.nih.gov/pubmed/22607208

13. Luo M, Jiang YL, Ma XX, Tang YJ, He YX, Yu J, Zhang RG, Chen Y, Zhou CZ. Structural and biochemical characterization of yeast monothiol glutaredoxin Grx6. J Mol Biol. 2010;398(4):614-22. Available from: https://europepmc.org/ abstract $/ \mathrm{med} / 20347849$

14. Li WF, Yu J, Ma XX, Teng YB, Luo M, Tang YJ, Zhou CZ. Structural basis for the different activities of yeast Grx1 and Grx2. Biochim Biophys Acta. 2010;1804(7): 1542-7. Available from: http://europepmc.org/abstract/MED/20417731

15. Tang Y, Zhang J, Yu J, Xu L, Wu J, Zhou CZ, Shi Y. Structure-guided activity enhancement and catalytic mechanism of yeast grx8. Biochemistry. 2014; 53(13):2185-96. Available from: https://pubs.acs.org/doi/abs/10.1021/bi401293s

16. Chi CB, Tang Y, Zhang J, Dai YN, Abdalla M, Chen Y, Zhou CZ. Structural and biochemical insights into the multiple functions of yeast Grx3. J Mol Biol. 2018; 430(8):1235-48. Available from: https://www.ncbi.nlm.nih.gov/pubmed/29524511

17. Szklarczyk D, Franceschini A, Wyder S, Forslund K, Heller D, Huerta-Cepas J, Simonovic M, Roth A, Santos A, Tsafou KP, et al. STRING v10: proteinprotein interaction networks, integrated over the tree of life. Nucleic Acids Res. 2015;43(Database issue):D447-52. Available from: https://www.ncbi.nlm. nih.gov/pmc/articles/PMC4383874/

18. Lee S, Kim SM, Lee RT. Thioredoxin and thioredoxin target proteins: from molecular mechanisms to functional significance. Antioxid Redox Signal. 2013;18(10):1165-207. Available from: https://www.ncbi.nlm.nih.gov/ pubmed/22607099

19. Coulter DA, Eid T. Astrocytic regulation of glutamate homeostasis in epilepsy. Glia. 2012;60(8):1215-26. Available from: https://www.ncbi.nlm.nih. gov/pubmed/22592998

20. Philpott CC, Leidgens S, Frey AG. Metabolic remodeling in Iron-deficient fungi. Biochim Biophys Acta. 2012;1823(9):1509-20. Available from: https:/ www.ncbi.nlm.nih.gov/pubmed/22306284

21. Yamaguchi-Iwai Y, Stearman R, Dancis A, Klausner RD. Iron-regulated DNA binding by the AFT1 protein controls the iron regulon in yeast. EMBO J. 1996; 15(13):3377-84. Available from: https:/www.ncbi.nlm.nih.gov/pubmed/8670839

22. Jacques JF, Mercier A, Brault A, Mourer T, Labbé S. Fra2 is a co-regulator of Fep1 inhibition in response to Iron starvation. PLoS One. 2014;9(6) Available from: https://www.ncbi.nlm.nih.gov/pmc/articles/PMC4045890/

23. Kumánovics A. Identification of FRA1 and FRA2 as Genes Involved involved in regulating the yeast iron regulon in response to decreased mitochondrial iron-sulfur cluster synthesis. J Biol Chem. 2008;283(16):10276-10286. Available from: https://www.ncbi.nlm.nih.gov/pubmed/18281282.

24. Pujol-Carrion N, Belli G, Herrero E, Nogues A, de la Torre-Ruiz MA. Glutaredoxins Grx3 and Grx4 regulate nuclear localisation of Aft1 and the oxidative stress response in Saccharomyces cerevisiae. J Cell Sci. 2006;119(Pt 21):4554-64. Available from: https://www.ncbi.nlm.nih.gov/pubmed/17074835

25. Dosztanyi Z, Csizmok V, Tompa P, Simon I. IUPred: web server for the prediction of intrinsically unstructured regions of proteins based on estimated energy content. Bioinformatics (Oxford, England). 2005;21(16): 3433-4. Available from: https://www.ncbi.nlm.nih.gov/pubmed/15955779

26. DeLano WL: Pymol: an open-source molecular graphics tool. 2002. Available from: http://www.pymol.org/ .

27. Krogh A, Larsson B, von Heijne G, Sonnhammer EL. Predicting transmembrane protein topology with a hidden Markov model: application to complete genomes. J Mol Biol. 2001;305(3):567-80. Available from: https://www.ncbi.nlm.nih.gov/pubmed/11152613

28. Abdalla M, Eltayb WA, EL-Arabey AA, Raihan M, Dafaalla TIM, Hamouda HI, Bhat EA, Awadasseid A, Ali HAA. Structure analysis of yeast glutaredoxin Grx6 protein produced in Escherichia coli. Genes Environ. 2018:40(15).

29. Thompson JD, Higgins DG, Gibson TJ. CLUSTAL W: improving the sensitivity of progressive multiple sequence alignment through sequence weighting, positionspecific gap penalties and weight matrix choice. Nucleic Acids Res. 1994;22(22): 4673-80. Available from: https:/www.ncbi.nIm.nih.gov/pmc/articles/PMC308517/

30. Kumar S, Stecher G, Tamura K. MEGA7: molecular evolutionary genetics analysis version 7.0 for bigger datasets. Mol Biol Evol. 2016;33(7):1870-4. Available from: https://www.ncbi.nlm.nih.gov/pubmed/27004904

31. Gauthier NP, Larsen ME, Wernersson R, de Lichtenberg U, Jensen $L$, Brunak $\mathrm{S}$, Jensen TS. Cyclebase.org - a comprehensive multi-organism online database of cell-cycle experiments. Nucleic Acids Res. 2008;36(Database issue):D854-9. Available from: https:/www.ncbi.nlm.nih.gov/pmc/articles/ PMC2238932/ 